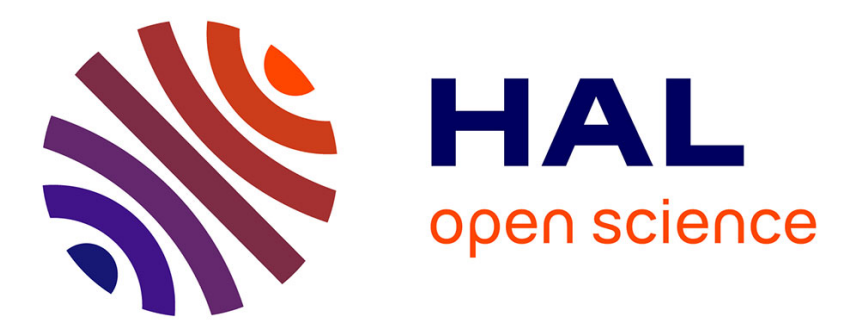

\title{
Les Veuves dans les textes de l'Angleterre du Moyen Age
}

Marie-Françoise Alamichel

\section{To cite this version:}

Marie-Françoise Alamichel. Les Veuves dans les textes de l'Angleterre du Moyen Age. Bulletin des Anglicistes Médiévistes, 2000, 58, pp.5-36. hal-01346339

\section{HAL Id: hal-01346339 \\ https://hal.science/hal-01346339}

Submitted on 18 Jul 2016

HAL is a multi-disciplinary open access archive for the deposit and dissemination of scientific research documents, whether they are published or not. The documents may come from teaching and research institutions in France or abroad, or from public or private research centers.
L'archive ouverte pluridisciplinaire HAL, est destinée au dépôt et à la diffusion de documents scientifiques de niveau recherche, publiés ou non, émanant des établissements d'enseignement et de recherche français ou étrangers, des laboratoires publics ou privés. 


\section{Marie-Françoise Alamichel \\ Université de Marne-la-Vallée}

\section{Les veuves dans les textes de l'Angleterre du Moyen Âge}

Les ouvrages portant sur la vie des femmes au Moyen Âge sont à la mode depuis de nombreuses années ; les féministes se sont emparées du sujet afin de montrer combien la moitié de l'humanité a toujours été maltraitée par l'autre moitié même si la condition féminine connut, selon elles, des périodes plus noires. Dans un but nullement universitaire, je m'intéresse depuis longtemps aux femmes de ma famille, à mes aïeules du XIX ${ }^{\mathrm{e}}$ siècle dont m'ont beaucoup parlé mes grands-mères et arrière-grands-mères. Et lorsqu'elles me racontaient la dure vie de leurs mères et grands-mères, je ne reconnaissais pas les schémas établis par le mouvement féministe depuis trente ans. Les créatures que l'on m'évoquait étaient de fortes femmes qui dirigeaient maison et commerce. Or, plusieurs avaient été veuves relativement tôt dans leur vie : était-ce là la raison de leur émancipation? Les multiples histoires des femmes consacrent peu de pages aux veuves. J'ai ainsi eu l'envie d'aller voir ce que les textes médiévaux anglais nous disaient d'elles. Je livre ici un premier aperçu d'une étude que je compte approfondir davantage par la suite.

Avant la révolution culturelle du $\mathrm{XII}^{\mathrm{e}}$ siècle, on ne s'intéressait guère aux relations entre hommes et femmes, aux sentiments. Les poèmes chantent essentiellement les exploits des guerriers, célèbrent l'honneur, le courage, la fidélité au chef et les femmes ne sont qu'entrevues dans les grand'salles. Les textes appartiennent aux hommes, leur donnent toute la parole : les femmes, veuves ou pas, sont quasi muettes, à l'exception de l'amante de The Wife's Lament. La littérature vieil-anglaise ne peut être, par conséquent, que de peu de secours dans une étude sur les veuves. Il s'agit, par conséquent, de se tourner vers d'autres documents. Ceux-ci (chroniques, homélies, etc) sont essentiellement le produit de clercs - des hommes qui, par leur fonction au sein de l'église, côtoyaient peu les femmes. On ne s'étonnera donc pas de ne rencontrer les personnages féminins, là encore, que très brièvement. En revanche, on constatera l'importance accordée dans la société aux veuves.

Pendant plus d'un siècle après l'arrivée des Normands, la rédaction en anglais disparut presque totalement et la littérature qui réapparut au XIII ${ }^{\mathrm{e}}$ siècle était radicalement différente, tant 
du point de la forme que du fond, de celle de la période vieil-anglaise. Dans l'Europe entière, de plus, le XII ${ }^{\mathrm{e}}$ siècle s'était intéressé à l'individu, l'intériorité, le moi et l'autre. On avait redécouvert certains auteurs grecs ou latins et l'Ars Amatoria d'Ovide inspirait grandement les troubadours et trouvères. Les poètes s'intéressèrent désormais aux femmes et à la question du mariage - voire du remariage. Ces textes, toutefois, ne sont pas des documents objectifs ; souvenons-nous de ce que Georges Duby écrivait au début de son étude des Dames $d u X I I^{e}$ siècle : «Pas plus que la sculpture ou la peinture, la littérature du XII ${ }^{\mathrm{e}}$ siècle n'est réaliste. Elle figure ce que la société veut et doit être. Reconstituer un système de valeurs, voilà tout ce qu'il m'est possible de faire ${ }^{1}$. Les veuves n'échappent pas à la règle : certes, les textes évoquent une certaine réalité, des attitudes, des coutumes, le voile et la robe noire mais ils reflètent avant tout la mentalité de l'époque, un mode de pensée codifié car les veuves intéressent principalement les moralistes et les sermonnaires. Et c'est pourquoi elles restent essentiellement un groupe impersonnel, une catégorie en un temps où les personnages féminins sortent peu à peu de l'ombre.

Les rares héroïnes de la littérature vieil-anglaise sont des femmes fortes, énergiques, aguerries. Judith (poème rédigé vers 930) est fondé sur le récit biblique mais l'auteur a traité l'histoire du meurtre d'Holopherne par Judith comme une épopée à l'atmosphère païenne et germanique, comme un chant patriotique. Judith, par son courage et par son autorité, a souvent été comparée à la reine de Mercie Æthelflæd, fille d'Alfred et redoutable guerrière. L'Elene de Cynewulf (Elene, $\mathrm{IX}^{\mathrm{e}}$ siècle) est présentée comme une reine martiale, une impératrice à la tête d'une armée, une puissante femme inflexible ${ }^{2}$. Ces femmes ne se distinguent guère des héros masculins de la poésie héroïque. Certes, cette dernière est loin d'épuiser l'abondance et la diversité de la littérature vieil-anglaise mais les autres genres représentés ont le même but de donner en modèle, de persuader, de juger, de mettre en garde. Les protagonistes sont des héros que les réalités de la vie quotidienne ne touchent pas : Julienne, par exemple, n'intéresse Cynewulf qu'en tant que martyre (Juliana, IX ${ }^{\mathrm{e}}$ siècle).

La Chronique anglo-saxonne ("éditée" pour la première fois en 891) ainsi que l'Histoire ecclésiastique du peuple anglais de Bède nous permettent d'entrevoir un court instant quelques

\footnotetext{
${ }^{1}$ Georges Duby, Dames du XII siècle, 1. Héloïse, Aliénor, Iseut et quelques autres, Paris : Gallimard, 1995, p 12. ${ }^{2}$ S. A. J. Bradley précise : «the portrait of the formidable Helen places her in that quite large gallery of women, literary and historical, recognized by the Anglo-Saxons as being in virtue, intellectual strength, vision, purpose and practical efficacy the peers or superiors of men ». S.A.J. Bradley, Anglo-Saxon Poetry, Everyman's Library, Londres : Dent, 1982, p 165.
} 
femmes anglo-saxonnes, et parmi elles un certain nombre de veuves. Les femmes que mentionne Bède sont toutes de rang royal - reines et princesses - ou des religieuses, de saintes abbesses entrées en religion dès leur plus jeune âge ou à la mort de leur mari. On ne s'attend pas à trouver chez Bède de nombreuses histoires de femmes ou de mariage/remariage. Or l'institution du mariage tient une place conséquente dans l'Histoire ecclésiastique ${ }^{3}$. En effet, l'évangélisation des Anglo-Saxons datait, pour le sud du pays, de la mission dans le Kent de saint Augustin (de Canterbury) en 597 et, pour le nord, de l'arrivée de moines irlandais dans l'île d'Iona en 563. L'Eglise se trouvait confrontée à des coutumes germaniques qui entraient en contradiction avec son enseignement. La Chronique anglo-saxonne précise qu'épouser la veuve de son père (il s'agit alors, bien évidemment d'une seconde épouse) pour les rois est l'habitude chez les païens germaniques :

616: Eadbald, son fils, lui succéda à la tête du royaume. Il abandonna le christianisme et suivit les pratiques païennes, prenant pour femme la veuve de son père. ${ }^{4}$

Bède nous donne plus de détails sur les raisons de l'interdiction d'épouser sa belle-mère. Æthelbert fut le premier roi du Kent à se convertir au christianisme suite à la mission de saint Augustin (597). Augustin apporta avec lui les interdits sexuels imposés par les Pères de l'Eglise latine - Tertullien (155?-220? $)^{5}$, saint Augustin [évêque d'Hippone] (354-430) et saint Jérôme (347?-420). Les Evangiles rappelaient les paroles prononcées par Adam sous l'inspiration divine au chapitre 2:24 de la Genèse : « N'avez-vous pas lu que le Créateur, au commencement, fit l'homme et la femme, et dit: C'est pourquoi, l'homme quittera son père et sa mère, et s'attachera à sa femme; et les deux ne feront plus qu'une seule chair? Ainsi donc, ils ne sont plus deux mais une seule chair. Que l'homme donc ne sépare pas ce que Dieu a uni » (Matthieu, 19:4-6). Saint Paul mentionne avec horreur un cas d'immoralité, un scandale commis dans la communauté des chrétiens de Corinthe : «On n'entend parler que d'une impudicité commise parmi vous, et si grave qu'on n'en rencontre pas de semblable chez les païens : c'est au point que l'un de vous vit avec la

\footnotetext{
${ }^{3}$ Voir A. Crépin, « Amour et mariage dans l'Angleterre du Haut Moyen Âge », Mariages à la mode anglo-saxonne, R. Lejosne \& D. Sipiere, éd., Presses de l'UFR CLERC, Université de Picardie, 1994, p 12-25.

${ }^{4}$ Pour le texte original, consulter J. M. Bately, éd., The Anglo-Saxon Chronicle, Ms A, Cambridge : D. S. Brewer, 1986 ou B. Thorpe, éd., The Anglo-Saxon Chronicle (Rerum Britannicarum Medii Aevi Scriptores 23), Londres : Longman, 1861, 2 volumes, vol. $\mathrm{n}^{\circ} 1$ : "Original Texts".

La loi de Moïse avait le même souci d'assurer une descendance aux défunts : "Maître, Moïse a prescrit: Si quelqu'un meurt, laissant une femme sans enfants, son frère épousera la femme et suscitera à celui-ci une postérité » (Luc, 20:28).

${ }^{5}$ Tertullien, s'étant placé hors de l'Eglise, eut moins d'influence que saint Augustin ou saint Jérôme. Il n'empêche que son Exhortation à la chasteté, aux nombreux passages misogynes ou qui dénoncent le mariage, était très connue au Moyen Âge : la femme de Bath des Canterbury Tales de Chaucer, par exemple, mentionne Tertullien au vers 676.
} 
femme de son père ! Et vous êtes enflés d'orgueil ! Et vous n'avez pas plutôt manifesté votre affliction, afin que soit retranché du milieu de vous l'auteur d'une telle action (...). Qu'un tel homme soit livré à Satan pour la destruction de son corps, mais afin que son âme soit sauvée au jour du Seigneur Jésus »(I, Corinthiens, 5:1-5). Bède reprend dans son Histoire ecclésiastique les questions adressées par Augustin, missionnaire inexpérimenté, au pape Grégoire le Grand. Parmi les neuf questions, la cinquième concerne le mariage : «Est-il permis à un homme d'épouser sa belle-mère ou sa belle-sœur ? ». Le pape répondit : «Épouser sa belle-mère est un grand péché, car la Loi dit: "Tu ne découvriras pas la nudité de ton père". Donc le fils ne peut pas découvrir la nudité de son père. Mais puisqu'il est dit, "Ils ne feront plus qu'une seule chair", quiconque se permet d'épouser sa belle-mère, qui n'était qu'une seule chair avec son père, commet par conséquent cette offense » (Hist. eccl. I, 27) ${ }^{6}$. Eadbald, fils d'Æthelberht de Kent, fut contraint de répudier sa belle-mère - et première femme - après sa conversion au christianisme : «Il renonça à son idolâtrie, renvoya sa femme illégitime, accepta la foi chrétienne et fut baptisé » (Hist. eccl., II, 6).

L'Église eut du mal à interdire de tels mariages qu'elle assimilait à de l'inceste. La pratique germanique avait pour but d'assurer une plus grande continuité aux dynasties, de limiter les rivalités entre les familles. Un siècle et demi après le règne d' Æthelberht, Æthelbald (frère d'Alfred le Grand) succéda à son père Æthelwulf et épousa en 858, sa veuve Judith - fille de Charles le chauve. Le précepteur et biographe d'Alfred, le Gallois Asser, nous fait part de son indignation :

A la mort du roi Æthelwulf, Aethelbald, son fils, violant l'interdit de Dieu et la dignité de chrétien, contrairement aussi à la pratique des païens ${ }^{7}$, s'empara du lit conjugal de son père et épousa Judith, fille de Charles, roi des Francs, s'attirant la réprobation de tous ceux qui eurent connaissance de cette affaire. Il régna sur le pays des West-Saxons pendant deux ans et demi ignominieux après son père. ${ }^{8}$

Bède nous rapporte que la conversion au christianisme d'Æthelberht par l'intermédiaire de saint Augustin fut grandement facilitée par le fait que l'épouse du roi, la reine Berthe, était déjà chrétienne (Hist. eccl. I, 25). L'influence religieuse et politique des reines, même veuves, est soulignée dans le texte de Bède et transparaît au début de la Chronique anglo-saxonne : au VIII ${ }^{\mathrm{e}}$ siècle, des femmes, appelées "reine" (regina) signent des chartes royales en tant que témoins, la

\footnotetext{
${ }^{6}$ On trouvera le texte original dans B. Colgrave et R. A. B. Mynors, éd. et trad., Historia Ecclesiastica Gentis Anglorum, Oxford : Clarendon Press, 1969.

7 Asser s'appuie ici sur l'Epître aux Corinthiens de saint Paul.

${ }^{8}$ Voir, W. H. Stevenson, éd., Asser's De Vita et Rebus Gestis Alfredi, Oxford : Clarendon Press, 1904.
} 
Chronique mentionne pour le Wessex la reine (cuen) d'Ine («722: cette année-là, la reine Æthelburh détruisit Taunton, ville qu'avait bâtie Ine »), la mort de Waerburh « reine de Ceolred » en 782, le mariage de Beorhtric et de Eadburh en 787 et précise « qu'en l'an 672, Cenwalh mourut et Seaxburh, sa reine, régna un an après lui ». Les références aux reines sont très nombreuses dans les chartes de la Mercie des VIII ${ }^{\mathrm{e}}$ et IX ${ }^{\mathrm{e}}$ siècles. On pense, bien évidemment, à Æthelflaed - "la Dame des Merciens" - fille du roi Alfred et mentionnée de multiples fois dans la chronique jusqu'en 918 où «elle mourut, douze jours avant le solstice d'été à Tamworth, dans la huitième année de son règne sur la Mercie en tant que seigneur légitime » ou encore à Ælfwynn : « en 919 la fille d'Aethelred, seigneur des Merciens, fut privée de toute autorité en Mercie : elle fut conduite dans le Wessex trois semaines avant Noël ». Avec le IX ${ }^{\mathrm{e}}$ siècle, les reines du Wessex disparaissent derrière la scène et ne sont que très rarement mentionnées. Asser nous explique d'ailleurs que Aethelwulf « ordonna que Judith, la fille du roi Charles qu'il avait reçue de son père, s'assoie à côté de lui sur le trône jusqu'à la fin de sa vie, bien que cela fût contraire à la coutume (injustifiée) de ce peuple. Car les West Saxons ne permettaient pas à la reine de s'asseoir à côté du roi, pas plus qu'ils ne permettaient qu'elle soit appelée "reine", mais seulement "la femme du roi" » (Asser, Vie du roi Alfred, § 13). Avec le IX ${ }^{\mathrm{e}}$ siècle, de plus, la chronique commence à être essentiellement consacrée aux mouvements et attaques des Vikings: les femmes n'ont aucune place dans ces activités guerrières. Un travail sur les veuves à l'époque anglo-saxonne est donc plus fructueux pour les VII ${ }^{\mathrm{e}}$ et VIII ${ }^{\mathrm{e}}$ siècles.

Bède mentionne plusieurs cas où des princesses chrétiennes mariées à des rois païens réussirent à convertir leurs maris et leurs peuples. A la même époque, des monastères féminins furent fondés en Angleterre et la vie conventuelle attira beaucoup de princesses et de reines. De nombreuses veuves royales devinrent abbesses. L'épisode le plus célèbre concerne la fondation de l'abbaye d'Ely en 673 avec Æthelthryth comme première abbesse. Celle-ci, pourtant mariée deux fois, réussit à demeurer vierge et obtint, très difficilement, de son second mari Ecgfrith, roi des Northumbriens, l'autorisation d'entrer au couvent. La seconde abbesse fut sa sœur, veuve du roi Ercenberht de Kent (Bède, Hist. eccl., IV, 19). En 718, à la mort du roi Ine de Wessex, on nous rappelle que sa sœur Cuthburh avait fondé « la communauté monastique de Wimborne. Elle avait été donnée en mariage à Aldfrith, roi de Northumbrie, mais ils se séparèrent de leur vivant ». Cette attitude était approuvée par l'Église à l'époque car Aldhelm (639-709), rédigeant un traité pour les 
religieuses de Barking fit l'éloge de Cuthburh, la louant d'avoir quitté son mari ${ }^{9}$. En l'an 800, Eadburh partit à la cour de Charlemagne à la mort de son mari, le roi Beorhtric de Wessex. Selon Asser, Charlemagne lui confia un grand monastère de femmes (on ne sait pas lequel) « dans lequel, ayant mis de côté les vêtements du monde temporel et revêtu ceux des moniales, elle remplit les fonctions d'abbesse - mais seulement pour quelques années » (Asser, Vie du roi Alfred, 15). Elle se révéla une fort mauvaise abbesse et mourut dans la pauvreté à Pavie après avoir été chassée de son couvent pour débauche. Au VIII' siècle, Eddius Stephanus écrivit en latin la biographie de l'évêque de York,Wilfrid. Celui-ci avait encouragé et aidé la reine Æthelthryth à quitter son mari pour devenir religieuse. Le roi, Egfrith, s'était alors remarié ; la nouvelle reine, Iurminburgh, était violemment hostile à Wilfrid mais, une fois veuve, elle devint, selon Eddius Stephanus, une très bonne chrétienne et, elle aussi, mère-abbesse d'un monastère :

Iurminburgh, la reine de Ecgfrith, jalousait beaucoup l'évêque Wilfrid. Par la suite, laissez-moi vous dire, après l'assassinat du roi, elle changea: elle passa d'une louve à un véritable agneau de Dieu, devint une abbesse parfaite à la tête de sa communauté. ${ }^{10}$

La pratique d'entrer au couvent pour les veuves - tout au moins pour les veuves de l'aristocratie car les textes ne parlent que de reines et de princesses - semble avoir été très courante au point que le code de lois que fit rédiger le roi d'origine scandinave Cnut (roi d'Angleterre de 1016 à 1035) comporte à l'article 73.3 la recommandation suivante : «Une veuve ne doit pas être consacrée religieuse trop précipitamment ».

Les codes de lois des rois anglo-saxons nous montrent que les veuves formaient une classe particulière, qu'elles avaient un statut légal spécifique. Æthelberht fut le premier roi à promulguer un recueil de lois en 602 qui établissait des "tarifs de composition" ou tarifs de Wergeld. Ces tarifs variaient selon la nature du délit, la qualité de la victime et la qualité sociale. Les lois d'Æthelbehrt distinguent quatre catégories de veuves et, par conséquent, quatre tarifs différents. Tout manquement à la tutelle exercée par un homme sur une noble veuve de la première classe coûte 50 shillings. Le tarif passe à 20, 12 et 6 shillings pour les veuves des trois classes suivantes. La différence entre une veuve de haute lignée et une veuve de la quatrième classe est donc presque

\footnotetext{
${ }^{9}$ Voir Aldhelm, On Virginity. M. Lapidge et M. Herren, éd., Aldhelm: The Prose Works, Totowa N. J. : Rowman and Littlefield, 1979.

${ }^{10}$ Voir B. Colgrave, Life of Bishop Wilfrid, Cambridge : University Press, 1927.
} 
d'un facteur neuf ${ }^{11}$.

Les codes de lois laissent entrevoir quelques aspects de la vie quotidienne des hommes et femmes de l'époque anglo-saxonne. Un petit nombre d'articles concernent les veuves. Les lois d'Hlothhere et de Eadric (qui régnèrent peut-être conjointement de 673 à 685) dans le Kent précisent à la clause 38 : « Si un homme meurt laissant femme et enfant, il est normal que l'enfant reste avec sa mère et l'un des parents paternels de l'enfant devra se porter volontaire comme tuteur pour s'occuper des biens de l'enfant jusqu'à ce que ce dernier ait dix ans ». Le recueil du roi Ine qui régna sur le Wessex de 688 à 726 donne quelques détails supplémentaires : «Si un homme et une femme ont un enfant ensemble et que le mari meurt, la mère garde l'enfant et l'élève. Elle se verra remettre six shillings pour s'occuper de lui, une vache en été, un bœuf en hiver; les parents proches doivent se charger de la demeure principale jusqu'à ce que l'enfant soit suffisamment grand » (clause 38). Le terme utilisé pour désigner le mari est ceorl dans le texte vieil-anglais, c'est-à-dire un paysan, un homme libre de classe inférieure. Le terme désignant l'enfant est bearn, il s'applique aussi bien à un garçon qu'à une fille (les testaments qui nous sont parvenus de l'époque anglo-saxonne montrent d'ailleurs que les filles héritaient de leurs parents, même si ce n'était pas toujours dans les mêmes proportions que leurs frères $)^{12}$. On constate dans ces deux exemples que si la veuve ne peut gérer les biens de son défunt mari, ses droits de mère ne sont pas contestés. Je travaille actuellement sur tout un ensemble de documents de l'époque anglo-saxonne (testaments, contrats de mariage, etc) pour voir, dans la réalité, comment ces lois germaniques des $\mathrm{VII}^{\mathrm{e}}$ et $\mathrm{VIII}^{\mathrm{e}}$ siècles étaient appliquées.

En 1008, Æthelred dit "the Unready", fit rédiger son propre code de lois. On y trouve deux articles consacrés aux veuves : «Et toute veuve qui se conduit bien sera placée sous la protection de Dieu et du roi » (21), «Et chaque veuve doit rester célibataire pendant douze mois ; ensuite, elle peut choisir ce qu'elle-même souhaite » (21.1). Cnut reprit, mot pour mot, ce second article dans

\footnotetext{
${ }^{11}$ F. Liebermann, éd., Die Gesetze des Angelsachen, Halle : Niemeyer, 1903, articles 75 et 75.1.

${ }^{12}$ Leo Carruthers a traduit le testament du IX ${ }^{\mathrm{e}}$ siècle d'Ælfred, duc de Surrey. On y découvre que le duc avait précisé : « Je fais librement don de mon héritage et de mes terres acquises par charte à mon épouse Werburgh et à notre enfant commune. (...) Moi, duc Ælfred, lègue, après ma mort, à Werburgh et à Audrey notre enfant commune, ce domaine [sus-mentionné], avec le bétail et les récoltes et tout ce qui s'y rattache, dont 2000 porcs avec les terres, à condition que Werburgh reste veuve selon notre accord verbal ». Le duc spécifie qu'il lègue 136 hides (1 hide = environ 60 hectares) à sa femme et sa fille tandis qu'il ne laisse que 3 hides à « [son] fils Æhelwold», héritier mâle, certes, mais illégitime. Leo Carruthers cite également le cas d'une femme qui, sous le règne de Cnut, déshérita totalement son fils au profit d'une de ses parentes. Leo Carruthers, L'Anglais médiéval, Paris, Turnhout : Brepols, 1996, p 72-3 et p 76-77.
} 
ses lois ajoutant ce qui devait incomber aux contrevenantes : «Et si elle choisit un mari dans l'année, elle se verra confisquer le morgengifu (= cadeau du matin) ${ }^{13}$ et toutes les possessions qu'elle avait obtenues de son précédent mari. Les parents les plus proches hériteront des terres et des biens quelle détenait avant » (73a). L'article suivant précise que la sanction sera identique en cas de mariage forcé, à moins que la femme ne soit d'accord pour quitter le nouveau mari. La clause 73.4 mentionne l'impôt que devait payer toute veuve, à la mort de son mari : le heriot, qui pouvait être une somme d'argent, mais était plus souvent la cession au seigneur de la plus belle tête de bétail : «Et chaque veuve a douze mois pour payer le heriot, sans pénalités, à moins qu'elle ne préfère payer plus tôt ${ }^{14}$. Enfin, l'article 74 rappelle que «ni une veuve, ni une demoiselle ne doit être forcée à épouser un homme qui lui déplaît, qu'elle ne doit pas être donnée pour de l'argent, à moins que l'homme ne choisisse de donner quelque chose volontairement ». Ces lois montrent que de telles habitudes existaient et qu'il était nécessaire de légiférer contre ces pratiques probablement courantes. Là encore, mon étude de documents est en cours. Les témoignages sont plus nombreux, les textes déjà fort connus pour l'époque d'après la Conquête : on y découvre que jusqu'au XIII ${ }^{\mathrm{e}}$ siècle, les riches veuves étaient des biens à monnayer par le roi et que l'Echiquier royal encaissa des sommes très importantes.

Contrairement à la littérature vieil-anglaise, celle de la période moyen-anglaise a beaucoup traité de l'alliance conjugale ${ }^{15}$. Il faut dire qu'à partir du $\mathrm{XI}^{\mathrm{e}}$ siècle, l'Église mit en place sa conception du mariage, en fit peu à peu un sacrement. Le concile de Latran de 1139 mit fin au mariage des prêtres et instaura une bénédiction des deux futurs époux devant la porte de l'église. C'est au XIII $^{\mathrm{e}}$ siècle (second concile de Latran en 1215) que l'on commença à exiger la publication des bans - moyen pour l'Eglise de vérifier s'il n'y avait ni lien de parenté proche ni bigamie - ainsi que le consentement mutuel des deux fiancés - droit essentiel qui devait protéger la femme occidentale. Concernant les veuves, la question qui nous intéresse est celle du remariage. La

\footnotetext{
${ }^{13}$ C'est-à-dire le cadeau donné, de l'argent ou des terres, une fois le mariage consommé. La femme gardait le morgengifu toute sa vie, c'était une sécurité en cas de décès du mari.

${ }^{14}$ En 1984, j'ai travaillé sur le village de Ramsey (Huntingdonshire), dominé par une très grande abbaye bénédictine au Moyen Âge. Mon travail portait sur l'architecture domestique. Le village, à cause de l'abbaye, est riche en archives. Le Liber Gersumarum de l'abbaye (document du $\mathrm{XV}^{\mathrm{e}}$ siècle) mentionne régulièrement les noms de veuves payant le heriot.

${ }^{15}$ Certains critiques voient dans la question du mariage l'un des thèmes structurants des Canterbury Tales de Chaucer, par exemple.
} 
position de l'Eglise, dès les tout débuts du christianisme, était ambiguë à ce sujet.

Saint Paul n'avait, en effet, pas tranché quant au statut des veuves. Dans l'épître aux Romains, on lit : «La femme en puissance de mari est liée par la loi à son mari toute la durée de sa vie ; mais si le mari vient à mourir, elle est dégagée de la loi qui la liait à lui. Si donc, du vivant de son mari, elle devient la femme d'un autre, elle sera taxée d'adultère ; mais si le mari meurt, elle est affranchie de la loi, en sorte qu'elle peut, sans être adultère, devenir la femme d'un autre » (Rom, 7:2-3). Par ailleurs, saint Paul avait aussi donné son avis personnel qui était le suivant : «La femme reste liée à son mari tout le temps de sa vie. S'il vient à mourir, elle est libre d'épouser qui elle veut, mais à la condition que ce soit dans le Seigneur. Toutefois, à mon avis, elle sera plus heureuse si elle demeure comme elle est. Et je crois bien avoir, moi aussi, l'Esprit de Dieu » (I, Cor. 7:39-40). L'Eglise primitive tâtonna : pour Tertullien, le remariage des veuves était interdit, mais le concile de Nicée (325) affirma le contraire tout en décourageant activement tout remariage. Au VI ${ }^{\mathrm{e}}$ siècle, le second concile de Braga condamna ceux qui se remariaient à faire pénitence pour lascivité. Ce jugement fut repris par plusieurs autres conciles et par les réformateurs du $\mathrm{XI}^{\mathrm{e}}$ siècle. Le droit canonique parvint à maturité au milieu du $\mathrm{XII}^{\mathrm{e}}$ siècle, l'ouvrage de base étant la Concordia discordantium canonum de l'Italien Gratien. Cet ouvrage, connu sous le nom de Decretum Gratiani (Décret de Gratien) devint l'un des recueils canoniques les plus usuels jusqu'à la publication du Code de droit canon....au XX $\mathrm{XX}^{\mathrm{e}}$ siècle ! L'ouvrage stipulait que les veuves pouvaient se marier autant de fois qu'elles voulaient et, reprenant saint Paul, précisait qu'il y avait véritablement un seul mariage valide tandis que les autres étaient tolérés à cause de la faiblesse humaine car «il vaut mieux se marier que de brûler» (I, Cor. 7:9). Le prologue du conte de la Femme de Bath nous montre qu'au XIV $\mathrm{XI}^{\mathrm{e}}$ siècle, la question restait d'actualité. La truculente Alison, mariée pour la première fois à 12 ans et qui se vante d'avoir eu cinq maris légitimes épousés «à la porte de l'église » (vers 6) connaît fort bien les textes bibliques patristiques. Elle commence par citer les Evangiles, les noces de Cana et la rencontre entre Jésus et la Samaritaine :

Figurez-vous qu'on m'a dit il n'y a pas longtemps que, comme le christ n'était allé qu'une seule fois à un mariage (c'était à Cana, en Galilée), qu'en donnant cet exemple, eh bien il avait voulu m'enseigner que je ne devais être mariée qu'une seule fois. Et puis aussi, écoutez comme Jésus, Dieu et homme, a été cassant le jour où il était assis à côté d'un puits et où il a fait ce reproche à la Samaritaine :

- Tu as eu cinq maris, lui a-t-il dit, et cet homme qui te possède maintenant n'est pas ton mari. 
Je vous assure que c'est ce qu'il a dit, ce qu'il voulait dire par là, cela, je ne puis le dire, sinon que je me demande pourquoi ce cinquième homme n'était pas le mari de la Samaritaine. Combien avait-elle le droit d'en avoir dans le mariage ? Je n'avais encore jamais entendu parler d'une définition de ce nombre. On peut faire des suppositions et retourner les interprétations dans tous les sens, mais moi, il y a une chose que je sais, c'est que Dieu nous a demandé de croître et de nous multiplier $^{16}$, je ne vous mens pas, et je suis tout à fait capable de comprendre ce noble texte. Je sais aussi fort bien qu'il a dit à mon mari qu'il devait abandonner son père et sa mère et se donner à moi ${ }^{17}$, mais sans mentionner de nombre, des histoires de bigamie et d'octogamie. Pourquoi alors aller parler de vilenie ? $^{18}$

Sur le ton comique de la fausse innocence, la femme de Bath reprend, en réalité, les arguments avancés par saint Jérôme dans l'Epistola adversus Jovinianum, arguments peut-être appris grâce à son cinquième mari qui appréciait la littérature anti féminine (voir à partir du vers 669). Le texte de Jérôme fut, tout au long du Moyen Âge, la référence de la littérature anti-matrimoniale.

En corollaire à la question du remariage des veuves, se posait le problème du délai à respecter entre deux mariages. Le droit romain interdisait aux veuves, mais pas aux veufs, de se remarier avant un an. Le droit canonique n'imposa aucune période minimum de veuvage. Le droit séculier, en revanche, reprit le délai d'un an : on a vu, par exemple, que les codes de lois d'Æthelred et de Cnut prescrivaient cette durée et mentionnaient des pénalités pour celles qui ne respecteraient pas la consigne. Le but premier de la clause était d'être sûr de la paternité d'un enfant né après la mort du mari. L'exemple de la Femme de Bath nous montre, qu'en littérature tout du moins, ce délai n'était pas obligatoirement respecté : la belle Alison remarque son cinquième mari le jour de l'enterrement de son quatrième époux puis « à la fin du mois l'amusant clerc Jeannot m'épousa en grande pompe ${ }^{19}$. La littérature et les documents historiques prouvent que le remariage était banal - surtout chez les très riches et les très pauvres. Matilda (1102-1167) était veuve de l'Empereur germanique Henry V depuis trois ans lorsqu'elle épousa Geoffroy Plantagenêt en 1128. Elizabeth Woodville (1437-1492) était veuve avec deux enfants lorsqu'elle rencontra, puis épousa, le roi Edouard IV. Margaret Beaufort (1443-1509), mère du roi Henry VII, fut mariée en 1450, à l'âge de sept ans, mais le mariage fut annulé en 1453. En 1455, elle épousa Edmund Tudor, demi-frère d'Henry VI. Elle fut veuve quelques mois plus tard, à treize ans. En 1464, elle épousa Sir Henry Stafford qui mourut en 1471. Thomas, Lord Stanley, fut son dernier mari à partir de 1473.

La célèbre correspondance de la famille Paston (The Paston Letters) écrite de 1420 à 1504

\footnotetext{
16 Genèse, 1 :28.

17 Matthieu, $19: 5$.

18 Geoffrey Chaucer, Les contes de Cantorbéry, J. Dor, trad., Paris : Christian Bourgois éditeur, collection 10/18, série «Bibliothèque médiévale », 1991, p 39-40.

19 Ibid., p. 54.
} 
couvre trois générations. Les Paston étaient de riches propriétaires terriens du Norfolk. On découvre ainsi Elizabeth (née en 1429), mariée à 29 ans et veuve seulement trois ans plus tard et qui se remarie à 42 ans. Edmond épousa successivement deux veuves : Catherine en 1480 et Margaret, déjà deux fois veuve, en 1491. L'histoire des Paston commence avec William et Agnès. Cette dernière fut veuve 35 ans et mourut de la peste en 1479. Or, son mari William était mort à 66 ans, soit un âge tout à fait respectable pour l'époque. William avait épousé Agnès à 42 ans. Ils eurent cinq enfants, nés entre 1421 et 1442. Comme en 1442 William avait 64 ans, il est bien évident que sa femme devait avoir, au moins, 20 ans de moins que lui. La correspondance nous montre que les hommes se mariaient relativement tard, vers 33/34 ans : John III, marié à 33 ans à Margery Brews, se remaria à 51 ans avec Agnes Morley, déjà deux fois veuve ${ }^{20}$. La littérature est pleine d'exemples où le mari est beaucoup plus âgé que la femme. Le vieux Janvier du conte du Marchand, «pendant soixante ans était resté célibataire. (...) Et assurément, aussi vrai que Dieu est roi, c'est chose glorieuse de prendre femme, surtout, lorsqu'on est vieux et chenu ; à ce moment, la femme devient le fruit de son trésor. C'est à cet âge qu'on doit prendre une jeune et jolie femme, de laquelle on pourra avoir un héritier $»^{21}$. Janvier s'adresse à ses amis et leur explique :

Mes chers amis, je vous préviens toutefois d'une chose. Je ne veux en aucun cas d'une vieille femme, et je veux aussi qu'il soit bien clair qu'elle ne doit pas avoir dépassé les vingt ans. J'ai envie de vieux poisson et de jeune chair. Le brochet est meilleur que le brocheton, et le veau tendre est meilleur que le vieux bœuf. Je ne veux pas d'une femme qui aurait trente ans, ce n'est qu'une tige de haricot séchée et du vulgaire fourrage. Quant aux vieilles veuves, bon Dieu, elles ont si bien appris l'art sur le bateau de Wade ${ }^{22}$ et savent si bien faire du mal quand l'envie leur en prend que je ne saurais jamais vivre tranquille avec elles. ${ }^{23}$

William Dunbar (1460?-1520?), poète écossais, écrivit une pièce comique intitulée Le Traité des deux femmes mariées et de la veuve. Les trois dames débattent sur le mariage, la première aimerait changer de compagnon tous les ans car elle est mariée à « une vielle chenille rampante » (vers 89), la seconde, inversement, préférerait un vieil homme à son noceur de mari devenu impuissant. Le premier mari de la veuve était un homme âgé et notre belle dame prit un amant, le second était un marchand d'âge mûr qu'elle mena à la baguette, qu'elle dépouilla de ses biens à son profit et à celui de son fils et qu'elle trompa allègrement ${ }^{24}$. William Langland

\footnotetext{
${ }^{20}$ N. Davis, éd., Paston Letters and Papers of the Fifteenth Century, Oxford : University Press, 2 vols., 1971, 1976.

${ }^{21}$ Geoffrey Chaucer, Les Contes de Cantorbéry, J. Dor, trad., références citées, p. 102-3.

${ }_{22}^{22}$ Figure légendaire obscure, probablement associée à la tromperie.

${ }^{23}$ Ibid., p. 106.

${ }^{24}$ J. Kinsley, éd., The Poems of William Dunbar, Oxford : University Press, 1979.
} 
(1331?-1390?) nous donne le point de vue du moraliste dans Pierre le Laboureur : «Ah par le Christ, comme il est répugnant de voir une jeune fille livrée à un vieil homme fatigué » (passus $\mathrm{IX})^{25}$. Le jeune âge de certaines des épouses explique en tout cas le nombre élevé de veuvages successifs que pouvait connaître une même femme, ainsi que nous le révèlent les documents historiques et les textes littéraires. On se doute des problèmes, d'ordre matériel ou affectif, des multiples querelles familiales que pouvaient entraîner ces mariages répétés : la littérature, les contes populaires en tête, témoignent des tensions que posaient l'existence de parâtres, de marâtres et d'enfants de différents lits. La veuve du Traité de Willam Dunbar se vante d'ailleurs d'avoir privilégié ses propres enfants au détriment de ceux d'un premier mariage de son mari :

J'ai élevé mes enfants comme des fils de Barons et ne me suis pas souciée du fretin de sa première femme. J'ai banni de mon territoire tous ses frères et j'ai toujours considéré ses amis comme mes ennemis, les haïssant (vers 402-5).

La pratique des mariages très précoces était l'apanage de la noblesse où elle était répandue. Entre 1288 et 1500, la grande famille des Berkeley compta cinq mariages dans lesquels chacun des deux époux avait moins de 11 ans. Un document anglo-latin rédigé pendant les années 1180, connu sous le nom de Rotuli de dominabus et pueris et puellis (Registre des riches veuves et des héritiers et héritières orphelins), recense pour la couronne royale les veuves et orphelins de la classe baronniale et précise leur richesse foncière. Les noms des veuves et demoiselles sont suivis de la mention in donatione Domini Regis («à donner selon la volonté du roi »). Quel était le rôle de ce registre? Les rois normands et angevins, à commencer par Guillaume le Conquérant, avaient octroyé des terres anglaises à leurs barons en récompense de services militaires et politiques. Les souverains voulaient s'assurer de la fidélité des héritiers de leurs vassaux et c'est ainsi que les riches orphelins devenaient tous leur pupille ou ceux d'un tuteur fidèle. Les orphelines, que l'on ne pouvait quand même pas déshériter, étaient mariées à des hommes dont le souverain était sûr. Au XII siècle, les rois préféraient une armée de mercenaires à des chevaliers de moins en moins enclins à faire la guerre. Ils vendaient donc la main des riches héritières ou des veuves fortunées. Le chroniqueur Matthew Paris relate le mariage de la Comtesse Margaret de Lisle qui eut pour conséquence d'unir « à un ignoble une noble, une femme pieuse à un impie, une belle à un vilain; elle lui avait été donnée, contrainte et forcée, par Jean le Tyran [Jean sans terre] qui ne répugna à

\footnotetext{
${ }^{25}$ W. W. Skeat, éd., Piers Plowman: Parallel-text Edition, Oxford : University Press, 1886.
} 
commettre aucun genre de crime $»^{26}$. Les ventes se faisaient aux enchères et la dame avait le droit de participer et d'acheter la possibilité soit de rester célibataire (il fallait payer davantage) soit de se choisir, elle-même, un mari. Mais les sommes étaient si importantes que plus d'une femme se serait alors vu contrainte de vendre son douaire et de perdre, par là même son moyen de subsistance. Le registre indique à chaque entrée l'âge des pupilles - une des veuves recensées n'a que dix ans $!^{27}$

Cette pratique était très impopulaire et fut dénoncée en 1215 dans la Grande Charte que dut signer Jean sans terre. Les articles 7 et 8 de la charte concernent les veuves de rang baronnial et stipulent que dorénavant elles conserveront les biens du foyer, n'auront rien à payer pour leur douaire et ne pourront plus être contraintes de se remarier ${ }^{28}$. La Grande Charte fut rédigée l'année où se tenait le second concile de Latran (on a vu que le consentement mutuel des deux fiancés était désormais obligatoire) : les deux entraînèrent des changements visibles.

Après la Grande Charte, le douaire fut de plus en plus souvent calculé sur les propriétés détenues par le mari au jour de sa mort et non au jour du mariage. A partir de 1300, de plus, la pratique de posséder les terres en commun se développa. Les deux combinés, de nombreuses veuves connurent une meilleure situation financière. Dans l'Angleterre anglo-saxonne, comme dans toutes les populations d'origine germanique, les femmes recevaient, au matin de la nuit de noces le morgengiefu, un présent (des terres ou des biens) offert par le mari dans l'intention de remercier son épouse du don de sa virginité, gage d'une paternité authentique. En cas de veuvage, un tiers restait à l'épouse, le reste retournait à la famille du défunt. Le Comte Godwine épousa la fille de Brihtric, sous le règne du roi Cnut. L'accord arrêté lors des fiançailles précisait qu'il « lui donnait une livre d'or et lui accordait la propriété à Street et toutes ses dépendances, 150 acres à Burmarsh et, en plus, 30 boeufs, 20 vaches, 10 chevaux et 10 esclaves ${ }^{29}$. On a vu que le code de lois de Cnut précisait que toute veuve se remariant dans l'année qui suivait le décès de son mari,

\footnotetext{
${ }^{26}$ Matthew Paris, Chronica Majora. Cité dans P. L'Hermite-Leclercq, L'Eglise et les femmes dans l'Occident chrétien, Turnhout : Brepols, 1997, p 269.

${ }^{27}$ J. H. Round, éd., Rotuli de Dominabus et Pueris et Puellis de XII Comitatibus, Londres, 1913.

${ }^{28}$ La Grande Charte fut rédigée en latin. Les liens entre l'Angleterre et la Normandie, perdue en 1204, étaient encore si forts qu'une version en français fut réalisée dès 1215. Elle est conservée à la Bibliothèque Muncipale de Rouen. Voici les articles 7 et $8:$ : La veve enpres la mort de son mari maintenant e sanz grevance ait son mariage e son heritage, ne riens ne doinst por son mariage ne por son doaire, ne por son heritage que ele e ses mariz tindrent al jor de la mort del mari, e seit en la maison de son mari puis qu'il sera morz xl. jorz, dedenz les quelsjorz li seit ses doaires livrez » (7) et « Nule veve ne seit destreite de sei marier tant dis come ele voldra vivre sanz mari, essi ne purquant que ele face seurté que ele ne se marira sanz nostre otrei,se ele tient de nos, o senz l'otrei de son seignor de qui ele tient, se ele tient d'autrui » (8). J. C. Holt, Magna Carta, Cambridge : University Press, 1965, p 250-1.

${ }^{29}$ Cité par K. Crossley-Holland, The Anglo-Saxon World, Oxford : University Press, 1982-4, p 261. Il s'agit ici de la dos, non du morgengiefu.
} 
serait privée de son morgengiefu. Le douaire supplanta progressivement la pratique du "cadeau du lendemain". Il s'agit, tout d'abord, d'une donation en pleine propriété, puis seulement en usufruit : ces biens que le mari assignait à sa femme pour en jouir en cas de veuvage représentaient environ un tiers de la fortune familiale. Une fois veuve, la femme gardait son douaire à vie - même si elle se remariait. Les femmes pouvaient donc accumuler plusieurs douaires. Chez William Langland, Pierre fait son testament, il ne s'agit donc pas de douaire que le mari annonçait le jour du mariage, à la porte de l'église. Mais la proportion habituelle du tiers est respectée - un tiers pour l'épouse, un tiers pour les enfants et un tiers pour le salut du testateur :

Je veux que ma femme reçoive tout ce que j'ai honnêtement gagné, et rien de plus. Elle le divisera entre mes chers fils et filles. Même si je devais mourir aujourd'hui, tout ce que je possède a été entièrement payé; tout ce que j'ai emprunté, je l'ai toujours rendu avant de rentrer chez moi. Quant à ce qui reste, je jure par la Croix de Lucques que je l'utiliserai pour honorer Vérité tant que je vis. ${ }^{30}$

L'auteur anonyme du $\mathrm{XIV}^{\mathrm{e}}$ siècle du poème Pureté cherche à montrer à chacun la voie de la perfection morale. Il recense les péchés humains en se fondant sur l'Epître aux Galates (5:19-21) et cite parmi les fautes, à côté du vol, de la convoitise, de l'excès de boisson ou du parjure, « déshériter et confisquer les douaires des veuves » $(\text { vers } 185)^{31}$. Le très grand nombre de procès portant sur le non respect des douaires montre que les veuves étaient, en effet, souvent lésées. Nos deux fortes personnalités que sont la Femme de Bath et la veuve du Traité de William Dunbar soulignent combien les questions économiques étaient essentielles aux yeux de ces deux intraitables femmes d'affaires : la Femme de Bath explique que «sur les maris que j'ai eus, trois étaient bons et les deux autres étaient mauvais. Les trois qui étaient bons étaient à la fois riches et vieux. (...) Ils m'avaient donné leurs terres et leur trésor, je ne devais plus m'évertuer à gagner leur amour ou à les respecter. Dieu du ciel, ils m'aimaient tellement que je n'appréciais plus leur amour. Une femme sage mettra toujours tout en œuvre pour obtenir l'amour de ceux qui ne lui en donnent pas. A part mon profit et mon plaisir, qu'avais-je encore besoin d'essayer de leur plaire, puisque je les avais bien en main et qu'ils m'avaient donné toutes leurs terres ? » ${ }^{32}$ La veuve du Traité des deux femmes mariées et de la veuve fait bien attention à obtenir des actes notariés lorsqu'elle dépouille son mari de ses propriétés :

\footnotetext{
${ }^{30}$ William Langland, Piers Plowman, références citées, passus VI.

31 A. C. Cawley \& J. J. Anderson, Sir Gawain and the Greeen Knight, Pearl, Cleanness, Patience, Londres : Dent, collection Everyman, 1962-1976, p 59.

${ }^{32}$ Geoffrey Chaucer, Les Contes de Cantorbéry, J. Dor, trad., références citées, p. 43.
} 
Lorsque tout fut sous mon commandement et qu'il m'obéit à la baguette, je criai sur ce lâche comme un coq lorsqu'il remporte la victoire. Lorsque je le vis servile et soumis alors je méprisai cet idiot et détestai ses manières. Alors je fus sans pitié, au point de vouloir lui faire souffrir le martyre. Je le harcelai comme une bête pour toute sorte de tâche puérile. Je l'aurais conduit à Rome, un licou autour de la tête, s'il n'y avait pas eu la question de ma réputation et du commérage des gens. Et, pourtant, je dissimulai toute ma haine dans mon cœur même si, parfois, celui-ci faisait de tels bonds que j'avais beaucoup de peine à le calmer. Toutefois je ne retirai jamais le bouchon de ma gorge tant que me manqua ce que je convoitai. Mais lorsque j'eus privé cet homme de ses biens terrestres, que j'eus obtenu ses maisons et ses propriétés foncières pour mon enfant, le bouchon s'échappa de ma gorge par vengeance si bien qu'il fut stupéfait de l'impact comme s'il s'était agi d'une arme d'acier. Moi, après une attente si longue, j'avais une telle soif de vengeance que j'étais aussi féroce qu'un dragon dans les querelles. J'avais feint si longtemps dans le but de flatter le sot- jusqu'au jour où mes titres de propriété furent scellés (vers 325-344).

Ces deux textes ont pour but de faire rire les lecteurs sur le thème bien connu du pauvre homme ridiculisé par son épouse qui porte la culotte dans le ménage. Pas de monde à l'envers chez William Langland qui vise à la satire sociale. Intelligence condamne ceux qui convoitent les veuves aisées comme cet « homme qui épouse, uniquement pour sa fortune, une riche veuve qui ne portera jamais d'enfant, si ce n'est dans les bras !» (passus IX) ou encore : «En vérité, mon frère, dis-je, ne vous comportez-vous pas comme l'un de ces prétendants qui ne cherchent à épouser une veuve que pour mettre la main sur leurs biens ? » (passus XI). Il est évident que les veuves riches étaient soumises à des pressions pour se remarier ce qui fait écrire à Claudia Opitz : «Certes, les veuves de la noblesse de la fin du Moyen Âge avaient une plus grande liberté de choisir leur époux que les "pucelles"; toutefois les possibilités de choix se limitaient à deux ou trois candidats proposés par la famille. Il était quasiment impossible à une veuve en âge de se remarier de prolonger ce temps de solitude - du moins lorsqu'elle possédait une fortune ou des terres $»^{33}$.

Les premiers travaux sur les femmes au Moyen Âge furent l'apanage de féministes qui affirmèrent trop vite que les veuves étaient plus libres que les femmes mariées, c'était mal connaître la société médiévale. Trop d'intérêts familiaux étaient en jeu avec les veuves aisées; en milieu urbain, les veuves d'artisans pouvaient continuer à travailler seules mais l'entreprise risquait de péricliter ce qui fait que ces femmes se remariaient dans une grande majorité, mais pas toujours ainsi que plusieurs études sur les veuves à Londres à la fin du Moyen Âge l'ont montré ${ }^{34}$. Quant aux

\footnotetext{
${ }^{33}$ G. Duby et M. Perrot, Histoire des femmes, vol. 2: "Le Moyen Âge", sous la direction de Christiane Klapisch-Zuber, Paris : Plon, 1990, p 324.

${ }^{34}$ C. Barron et A. Sutton, Medieval London Widows 1300-1500, 1994.

K. Lacet, « Women and Work in Fourteenth and Fifteenth Century London », in L. Charles et L. Duffin, éd., Women and Work in Pre-Industrial England, Beckenham, 1985.
} 
pauvres veuves des campagnes, tout les poussait à se remarier car il était difficile de faire face, seule, à tous les travaux des champs. Henrietta Leyser cite même le cas de Launton (Oxfordshire) où une veuve ne pouvait pas garder sa terre plus d'un an et un jour si elle ne se remariait pas. Si elle restait célibataire plus longtemps, elle devait se contenter de son douaire (un tiers des terres) ${ }^{35}$. Dans les Contes de Canterbury de Chaucer, les veuves vivant à la campagne semblent misérables ${ }^{36}$. L'adjectif poor s'accole à wydwe comme épithète de nature : la proie du semoneur dans le Conte du Frère est « une veuve âgée, une vieille » (1377) qui explique qu'elle ne peut pas payer douze pence car elle est « vieille et pauvre » (1608), la propriétaire de Chantecleer est :

Une pauvre veuve d'un âge assez avancé; elle vivait dans une étroite chaumière, près d'un petit bois, au fond d'un vallon. Depuis le dernier jour de sa vie d'épouse, cette veuve, dont je vais vous raconter l'histoire, menait patiemment une vie toute simple. Elle n'avait guère de biens ni de rentes et subvenait à ses besoins et à ceux de ses deux filles en gérant avec économie ce que Dieu voulait bien lui envoyer. Elle avait trois grosses truies, pas davantage, trois vaches, et aussi une brebis du nom de Maud. Ses appartements privés et sa salle à manger, dans laquelle elle faisait plus d'un maigre repas, étaient tous les deux noirs de suie. Elle ne risquait pas d'avoir besoin de sauce piquante, aucun morceau délicat ne lui passait en effet par la gorge.

Pour conclure sur la question de la liberté des veuves, on peut cependant dire qu'elles connurent leur plus grande "liberté" entre 1300 et 1500 : elles avaient obtenu « d'administrer librement les biens provenant de leur dot et de leur douaire et de n'avoir recours à un intercesseur masculin que dans les cas de différends ou dans les actes administratifs importants. (...) En Italie, dès le XIVe siècle, dans le reste de l'Europe environ cent cinquante ans plus tard, la tutelle exercée par les hommes sur les femmes fit son retour dans la législation $»^{38}$.

Les riches veuves qui ne souhaitaient pas se remarier pouvaient choisir d'entrer dans l'une des communautés religieuses bénédictines, cisterciennes ou, plus tard, dominicaines. Ces couvents étaient réservés aux nobles dames fortunées qui payaient cher l'entrée dans ces communautés opulentes. L'Église avait toujours encouragé le veuvage chaste. Dans le poème de G. Chaucer, Troilus et Criseyde, Criseyde, jeune et riche veuve, explique à son oncle qui insiste pour qu'elle ne

\footnotetext{
35 Henrietta Leyser, Medieval Women, Londres : Weidenfeld \& Nicolson, 1995. Edition de poche, Londres : Phoenix, 1996, p 180 .

${ }^{36}$ Hélène Taurinya Dauby, Le Rôle social de la femme d'après The Canterbury Tales de Chaucer et le Menagier de Paris, Paris : Publications de l'AMAES, 1985, p 62-73.

37 Geoffrey Chaucer, Les Contes de Cantorbéry, J. Dor, trad., références citées, p 198.

${ }^{38}$ Claudia Opitz in G. Duby et M. Perrot, Histoire des femmes, références citées, p 325 et p 328.
} 
porte plus ses habits noirs et son voile et pour qu'elle aille danser : «Oh, Dieu m'en garde! dit-elle, êtes-vous fou? Est-ce là une vie pour une veuve, que Dieu vous protège ! Mon Dieu, vous m'effrayez ! Vous agissez si bizarrement qu'on dirait que vous divaguez. Il serait beaucoup plus convenable pour moi de toujours être à prier et de lire des vies de saints dans une grotte » (Livre II, vers $113-7)^{39}$. Criseyde fait ici allusion à une vie pieuse de veuve calquée sur les dernières années (légendaires) de Marie-Madeleine. La première Epître de saint Paul à Timothée indiquait ce que l'on attendait alors des veuves :

Honore les veuves, celles qui le sont véritablement. Si une veuve a des enfants ou des petits-enfants, qu'elle leur apprenne avant tout à témoigner la piété filiale envers leur propre famille et à rendre à leurs parents ce qu'ils en ont reçu car cela est agréable à Dieu. La vraie veuve, celle qui reste seule au monde, met son espérance en Dieu, et ne cesse de vaquer, nuit et jour, à la prière et à l'oraison. Celle au contraire qui vit dans les plaisirs est morte, toute vivante qu'elle paraisse. Rappelle-leur cela afin qu'elles soient irréprochables (I, Cor. 5:3-7).

Les textes montrent que l'idéal de vie prôné par les clercs et moralisateurs pour les veuves était la continence. Dès la période anglo-saxonne, chasteté et veuvage étaient associés : Asser nous explique que la belle-mère du roi Alfred, Eadburh, « était une femme remarquable qui resta, de nombreuses années après la mort de son mari, une chaste veuve » (chapitre 29). Dans le premier quart du XIII ${ }^{\mathrm{e}}$ siècle fut rédigée en Angleterre la série de textes connue sous le nom de groupe de sainte Catherine qui comporte, entre autre, l'épître sur la Virginité sacrée (Hali Meiðhad), qui reprend tous les arguments de la littérature anti-matrimoniale de l'époque et de ses sources patristiques. La virginité y est présentée comme l'idéal de vie des femmes chrétiennes qui deviennent ainsi, selon la formule traditionnelle, les épouses de Dieu : «Zion était jadis le nom d'une haute tour de Jérusalem (...). Et cette tour symbolise la position éminente de la virginité, qui, comme depuis un sommet, voit toutes les veuves en bas et les femmes mariées aussi. Car celles-ci, en tant qu'esclaves de la chair, sont enchaînées au monde et vivent sur les basses terres. Et la vierge demeure, grâce à sa vie transcendante, dans la tour de Jérusalem » ${ }^{40}$. Cette hiérarchie se double de tarifs :

De ces trois états - la virginité, le veuvage et le mariage qui arrive en troisième - on peut dire, par leur degré de béatitude lequel est supérieur aux autres et de combien. Car le mariage est récompensé

\footnotetext{
${ }^{39}$ Geoffrey Chaucer, Troilus and Criseyde in L D. Benson, ed., The Riverside Chaucer, Oxford : University Press, 1987.

${ }^{40}$ B. Millett et J. Wogan-Browne, Medieval English Prose for Women, Oxford : Clarendon Press, 1990.
} 
trente fois au Ciel, le veuvage soixante fois et la virginité, qui les surpasse tous les deux, cent fois. Cette formule était extrêmement courante au Moyen Âge et découlait directement de la parabole du semeur de l'évangile de saint Matthieu : «D'autres [graines] enfin tombèrent dans la bonne terre : elles donnèrent du fruit, cent pour une, soixante pour une, trente pour une » (13:8). Saint Jérôme emprunta cette parabole et l'adapta pour son discours sur le mariage. C'est ainsi qu'il écrivit à Eustochium :

Ta sœur Blésilla, ton aînée par l'âge, mais ta cadette dans la profession religieuse, au bout de sept mois de mariage est devenue veuve. O condition humaine vouée au malheur et ignorante de l'avenir ! Elle a donc perdu et la couronne de la virginité et la volupté du mariage. Elle observe, bien entendu, le second degré de la chasteté. Mais n'imagines-tu pas quelles croix elle supporte par moments, alors que chaque jour elle admire en sa sœur ce qu'elle a perdu, alors que, si difficile qu'il soit de se passer de la volupté jadis goûtée, elle ne saurait attendre de sa continence qu'une récompense moindre? Qu'elle soit pourtant confiante! Qu'elle soit joyeuse ! Le fruit cent fois multiplié ou celui qui l'est soixante fois seulement proviennent d'une même semence : la chasteté. ${ }^{41}$

La littérature hagiographique, les sermons, les traités de morale religieuse ont véhiculé, répété, développé cette répartition de la gent féminine en trois catégories qui ne laisse aucune place à l'individualisation: les femmes sont classées dans l'un ou l'autre des états et tout semble alors avoir

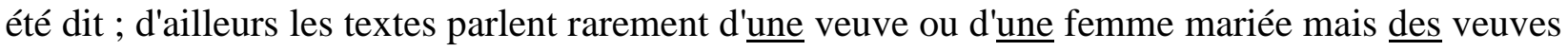
ou des femmes mariées. Geoffrey Chaucer dans le prologue du Légendier des Dames vertueuses (écrit vers 1386) précise qu'il va raconter la vie de dames vertueuses pour se faire pardonner de ses positions antiféministes soutenues dans Troilus et Criseyde. Le dieu de l'amour, qui le critique, lui rappelle qu'il a dans sa bibliothèque le traité de saint Jérôme qui donne l'exemple d'une bonne centaine de vierges qui furent pures, d'épouses qui restèrent fidèles et de veuves qui furent constantes toute leur vie: les femmes sont, ici aussi, réparties selon les trois états habituels. ${ }^{42}$

Cette hiérarchie entre vierges, veuves et femmes mariées a donné lieu à plusieurs séries

\footnotetext{
${ }^{41}$ Jérôme, Lettres, texte établi et traduit par J. Labourt, Paris : Les Belles Lettres, 1951, tome II, p 124. Ælfric, au X $\mathrm{X}^{\mathrm{e}}$ siècle, reprit la formule de Jérôme. Voir son homélie intitulée In Purificatione Sanctae Mariae (le texte est en vieil-anglais) dans P. Clemoes, éd., Aelfric's Catholic Homilies, the first series, EETS, Oxford : University Press, 1997, p 255.

${ }^{42}$ Geoffrey Chaucer, The Legend of Good Women in L. Benson, éd., The Riverside Chaucer, références citées, vers $282-5$, p 597.

«In most kinds of medieval literature, women are seen principally in their sexual roles and are defined in sexual terms. Whether as virgins, wives, or widows, women are seen by men, even by presumably chaste men such as preachers, in the light of that which makes them most different from men. They are therefore defined as being either presexual, sexually active or postsexual », Leo Carruthers, « No Womman of no clerk is preysed », A Wyf ther was, J. Dor, éd., Liège: L3, 1992, p 51.
} 
d'images. Jérôme compare les premières à des lis, les secondes à des violettes et mentionne les roses des martyres dans sa lettre à la veuve Furia. Hali Meiðhad préfère les pierres précieuses : «Bien que tu sois une vierge, ne minimise pas les veuves et les femmes mariées. Car de la même façon qu'un rubis est intrinsèquement plus précieux qu'une jacinthe et qu'une jacinthe brillante est toutefois plus précieuse qu'un rubis terne, une demoiselle, par la vertu de la virginité est supérieure aux veuves et aux femmes mariées; et, pourtant une épouse humble ou une veuve douce est supérieure à une vierge hautaine». William Langland a choisi l'image des fruits d'un arbre splendide, Charité, pour rendre cette classification dans Pierre le laboureur :

Là, tout en bas, répondit-il si le besoin s'en fait sentir, je peux cueillir le fruit du mariage : c'est un bon fruit juteux. Un peu plus haut pousse le fruit de la Continence qui est aussi bon qu'une délicieuse poire de Cailloux. Mais les plus hautes branches engendrent le fruit le plus achevé, à la saveur la plus raffinée: le fruit de la virginité qui égale celui des anges. C'est lui qui arrive à maturité le premier, se gorge de sucre sans gonfler et, jamais, ne devient aigre (passus XVI).

Quelques vers plus loin, Foi propose une autre métaphore filée en comparant les trois états à la Trinité : « Les états de mariage, veuvage et de virginité, qui symbolisent la Trinité, descendent tous d'un seul être humain, Adam notre père à tous. (...) Le mariage représente la puissance de Dieu puisqu'il multiplie la race humaine. Et, si je puis dire, c'est un excellent symbole pour le Père Céleste qui est à l'origine de tout. Le Fils, pour continuer avec cette image hardie, correspond clairement à la veuve: "Mon Dieu, Mon Dieu, pourquoi m'as-tu abandonné ?" Ces paroles montrent que le Créateur devint une créature afin de savoir ce qu'étaient l'un et l'autre. Mais de même qu'il ne pouvait y avoir de veuve sans mariage, de même Dieu ne pouvait devenir homme sans avoir une mère. Assurément, une veuve qui n'a pas été mariée est un non-sens, de même un mariage sans enfants mérite à peine ce nom » (passus XVI). John Gower (1330?-1408), quant à lui, compare nos trois états à trois des cinq filles (Bonnegarde, Virginité, Matrimoine, Continence et Aspre Vie) de Dame Chasteté dans son long poème écrit en français Mirour de l'Omme. Parmi les disciples de Continence, on trouve les frères, les moines, les religieuses qui «sont obligez a contenir » mais «la dame auci qui voet tenir/ Sa chasteté, dont revestir/ Se fait d'anel par beneiçoun / D'evesque, apres pour nul desir / Se porra lors descontenir, / Si trop ne passe sa resoun $»(\text { vers } 17827-32)^{43}$.

${ }^{43}$ G. C. Macaulay, éd., The Complete Works of Gower, Oxford : Clarendon Press, 1899-1902. 
Il existait, en effet, au sein de l'Eglise, un ordre particulier : l'ordre des veuves ${ }^{44}$. Les femmes vivant dans l'entourage de Jésus étaient nombreuses, probablement des veuves ou des femmes séparées car elles sont présentées comme indépendantes, libres de leurs personnes et de leurs biens. Elles sont mentionnées dans les Evangiles - Marie-Madeleine, Marie (mère de Jacques et de Joseph), la mère des fils de Zébédée, Marie Salomé, Jeanne, Suzanne, etc - et au début des Actes. L'institution de l'état de veuvage rayonna bientôt en dehors de Jérusalem : à Joppé, peut-on lire, vivait une femme nommée Tabitha «qui se consacrait aux bonnes œuvres et distribuait de nombreuses aumônes »; celle-ci venant de mourir, Pierre se rend à Joppé et «à son arrivée, on le conduisit dans la chambre haute et il y trouva toutes les veuves » (Actes, 9:36 et 39). La première Epître à Timothée atteste déjà l'existence d'un ordre des veuves : «Pour être inscrite sur le rôle, il faut qu'une veuve ait au moins soixante ans, et qu'elle n'ait été mariée qu'une fois, que sa bonne conduite lui ait créé une bonne réputation, qu'elle ait bien élevé ses enfants, exercé l'hospitalité, lavé les pieds des saints, secouru les malheureux, pratiqué toute espèce de bonnes œuvres (I, Tim., 5: 9-10). L'existence de cet ordre des veuves nous est confirmée par la littérature ecclésiastique archaïque. Ignace (mort vers 110) parle des «vierges appelées veuves », ce qui montre que le mot "veuve" a ici le sens d'un ordre ecclésiastique ${ }^{45}$. A partir du III $^{\mathrm{e}}$ siècle, l'ordre des vierges eut tendance à prendre une place plus importante que celui des veuves ; par ailleurs, apparurent, au milieu du $\mathrm{III}^{\mathrm{e}}$ siècle, les diaconesses qui se substituèrent aux veuves.

$\mathrm{Au} \mathrm{XII}{ }^{\mathrm{e}}$ siècle, un renouveau religieux se fit jour. Une nouvelle attitude se dessina : le souci fut désormais d'imiter le Christ par la pauvreté et la charité, de retourner aux vraies valeurs de la vie chrétienne. Les évangiles furent relus comme des documents historiques, des règles de vie. Pierre Abélard, par exemple, après les multiples attaques essuyées lors de l'installation d'Héloïse et de ses compagnes au Paraclet, écrivit à un ami : «Mes ennemis, s'ils avaient vécu au temps du Christ et de ses membres, les prophètes, les apôtres, les Saints-Pères, ne leur auraient pas épargné leurs calomnies, en les voyant, sans pourtant la moindre impureté, vivre dans la familiarité des femmes ! Saint Augustin, dans son livre sur l'Oeuvre des moines, note que les femmes s'attachèrent à Notre-Seigneur et aux apôtres, et devinrent leurs inséparables compagnes, au point de les suivre

\footnotetext{
44 Je remercie Leo Carruthers pour ses explications très éclairantes sur cet ordre.

${ }^{45}$ Cité par J. Daniélou et H. Marrou, Nouvelles Histoire de l'Eglise, Des Origines à saint Grégoire le Grand, Paris : Seuil, 1963, p 149.
} 
même dans leurs tournées de prédication ${ }^{46}$. Au XII ${ }^{\mathrm{e}}$ siècle, de nombreux observateurs, Jacques de Vitry en tête, notèrent le nombre très important de femmes qui se consacraient à la vie religieuse : moniales mais aussi recluses, béguines. Les veuves qui ne souhaitaient pas se remarier, mais qui ne voulaient pas entrer au couvent, pouvaient faire vœu de chasteté. La cérémonie était publique : l'évêque bénissait la femme et lui remettait un anneau et une mante, «anel par beneiçoun d'evesque » nous a dit John Gower. Jacques de Vitry raconta l'histoire de Marie d'Oignies ${ }^{47}$ qui mourut en 1213 : née dans une famille aisée, mariée à 14 ans, la jeune femme réussit à persuader son mari de vivre dans la continence et de transformer leur propriété en léproserie. Marie mena une vie de grande austérité et de sainteté. Sa renommée, qui attirait beaucoup de visiteurs, la décida à se retirer et à vivre en ermite dans une cellule à côté du monastère d'Oignies où elle mourut. Le texte de Jacques de Vitry fut traduit en moyen-anglais au XIVe siècle ${ }^{48}$. Marie d'Oignies - cette épouse du Christ - est présentée comme ayant de nombreuses visions ; « une autre fois, lorsqu'une veuve consacrée $^{49}$ qui avait longtemps servi Dieu dans le saint ordre des veuves et qui avait maintenu ses filles dans la sainte virginité pour l'époux céleste, fut sur son lit de mort à Villambroc près de Nivelle, elle [= Marie d'Oignies] vit Notre Dame debout à côté de la veuve bénie ».

Margery Kempe (1373?-1440?) cite l'histoire de la vie de Marie d'Oignies au chapitre 62 de son autobiographie. C'est en 1413, après avoir eu quatorze enfants, qu'en accord avec son mari, Margery fit vœu de chasteté. Au chapitre 15 de son livre, elle relate leur demande à l'évêque de Lincoln, insistant sur l'anneau et la mante qu'elle doit recevoir. Tout au long du livre, on découvre que Margery entretint un dialogue nourri avec le Christ qui l'appelle «mon amante, mon épouse bienheureuse, ma sainte femme » (chapitre 86$)^{50}$, lui explique qu'il a « retiré l'amour de son cœur de tous les cœurs des hommes pour le mettre dans son cœur » (chapitre 65) et ajoute qu'elle a de multiples raisons de l'aimer plus que tout en particulier : «parce qu'[elle] a ce qu'elle voulait en matière de chasteté, comme si elle était veuve, alors que son mari est toujours vivant et en bonne santé » (chapitre 65). Le Livre de Margery Kempe désigne la vie comme un veuvage inversé : le mari, le Christ, est perdu avant de pouvoir être rejoint pour des noces mystiques. Le vocabulaire

\footnotetext{
${ }^{46}$ Abélard et Héloïse, Correspondance, texte traduit et présenté par P. Zumthor, Paris : Union générale d'éditions, Collection 10/18, série Bibliothèque médiévale, p 107.

${ }^{47}$ Jacques de Vitry, Vita Maria Oigniacensis, in Acta Sanctorum, 1867, n²5, p. 542-72.

${ }^{48}$ C. Horstmann, éd., « Prosalegenden: Die Legenden des ms. Douce 114 », Anglia, 1885, n8, p 102-96.

${ }^{49}$ Le texte moyen-anglais dit: « a religyous widowe ».

${ }^{50}$ S. B. Meech et H. E. Allen, TheBook of Margery Kempe, Oxford : University Press, EETS n²12, 1940.
} 
utilisé pour dépeindre l'amour, pas encore comblé, de la mystique est celui du deuil : Margery pleure son amant céleste et craint, ayant été mariée, de ne pouvoir danser au ciel avec les vierges. Le Christ la rassure, elle le rejoindra car «[elle] est une demoiselle dans [son] âme » (chapitre 22), il la prendra par une main, sa mère la prendra par l'autre et alors elle pourra danser et il lui dira : «Bienvenue dans mon royaume de joie et d'allégresse pour que tu demeures avec moi et que, jamais, tu ne sois séparée de moi » (chapitre 22). Il reste à Margery quinze ans de veuvage sur terre - ce que, nous dit-on, connut avant elle, Marie-Madeleine qui survécut quinze ans à Jésus (chapitre 74). Au bout des quinze ans, Margery ira vivre éternellement auprès de sainte Marie (la Sainte Vierge), sainte Catherine, sainte Marguerite (deux vierges martyrisées), sainte Marie-Madeleine (la pécheresse repentie), les apôtres, les martyrs, les confesseurs, les vierges bénies (chapitre 86).

Cette liste n'a rien d'original, on la trouve dans de nombreux textes religieux, à l'instar de Hali Meiðhad ou de Sawles Warde (La Garde de l'âme), autre texte du Groupe de sainte Catherine. William Langland se distingue en écrivant, dans les trois versions de Pierre le laboureur, que siègent au ciel saint Jean, saints Simon et Jude, «les vierges, les martyrs, les confesseurs et les saintes veuves » (passus XII). Cette dernière expression, The holi widowes, est surprenante car dans l'état actuel de mes recherches - je ne l'ai trouvée nulle part ailleurs. Il s'agit à l'évidence des saintes femmes, premiers témoins de la résurrection du Christ. Mais les autres textes que j'ai consultés et qui les mentionnent ne les appellent jamais veuves : on trouve dans les pièces de la passion «les femmes » ou «les trois Marie », dans les textes hagiographiques comme The South English Legendary ${ }^{51}$ «the holy maide» pour les vierges et «this holy womman » pour Marie d'Egypte ou Marie-Madeleine. Marthe est appelée «god womman ». Aucune des trois n'est qualifiée de « sainte veuve ». Il me semble difficile d'admettre que William Langland ait été le seul à utiliser cette formule : cette étude se termine donc sur un point d'interrogation que je souhaite pouvoir effacer le plus rapidement possible.

Au terme de ce travail, je constate que les textes que j'ai abordés parlent bien peu de sentiments, de douleur, d'absence. Les veuves ne pleuraient-elles pas celui qu'elles avaient perdu tous les mariages n'étant pas, bien évidemment, de simples contrats économiques? La littérature

51 C. d'Evelyn \& A. J. Mill, The South English Legendary, Oxford : University Press, EETS n²35, 1956. 
médiévale anglaise a ceci de paradoxal que seules deux hypocrites nous montrent le comportement attendu d'une femme éprouvée par la perte de son époux. La veuve joyeuse de Dunbar (Traité des deux femmes mariées et de la veuve) nous explique qu'elle fait semblant d'être désespérée :

Je pleure comme si je souffrais mais je suis désormais heureuse. Je m'habille comme si je pleurais mon mari mais mon cœur est léger. Ma bouche parle de tristesse et mon esprit rit. Mes manteaux indiquent l'affliction avec leur couleur noire mais mon corps, par en-dessous, est raffiné et habillé avec soin. Je baisse la tête et semble l'image du désespoir dans mes habits de deuil comme si les hommes n'étaient plus jamais pour moi.

Lorsque je vais à l'église, vêtue de noir, je compose mon expression comme un renard dans une toison d'agneau (...) Lorsque des parents de mon mari me voient au loin, j'ai une éponge mouillée, pour le chagrin, caché dans mon ample manteau. Et, par moments, je la presse avec astuce et me mouille les joues afin que mes yeux pleurent et que les larmes coulent (415-23 et 436-9).

La Femme de Bath avait adopté la même tactique après la mort de son quatrième mari : «je n'arrêtai pas de pleurer et de faire triste mine, comme doivent le faire les épouses parce que l'usage le veut $»^{52}$. Mais la belle repère les jambes d'un des porteurs du cercueil, de si belles jambes qu'elle donne son cœur à celui qui les possède ! Le paradoxe va plus loin car les deux seuls êtres, que j'ai recensés, qui pleurent sincèrement leur conjoint sont, non pas des veuves, mais deux veufs : le premier est Sir Orfeo (Sir Orfeo, lai du $\mathrm{XIV}^{\mathrm{e}}$ siècle) qui apprend que son épouse va lui être enlevée et se lamente, «j'aimerais mieux perdre ma vie que de perdre ainsi la reine ma femme » puis qui la cherche pendant dix années de chagrin et de mélancolie ${ }^{53}$. Le second est John of Gaunt qui pleure la mort de sa femme Blanche (décédée en 1369) dans le poème de Geoffrey Chaucer, Le Livre de la Duchesse. Aucun auteur n'a su mettre dans la bouche d'une femme les paroles d'amour que Chaucer prête au Duc : «Je suis plongé dans la plus profonde des peines / Je n'ai plus aucune joie ni aucun plaisir / Maintenant que je sais que ma belle Dame / Que j'ai aimée de tout mon cœur / Est loin de moi, morte, partie» (vers 475-9). On ne peut, une fois de plus, que regretter de ne pouvoir ressusciter la parole des femmes.

${ }^{52}$ Geoffrey Chaucer, Les Contes de Cantorbéry, J. Dor, trad., références citées, p 53.

${ }^{53}$ K. Sisath, éd., Fourteenth Century Verse \& Prose, Oxford : Clarendon Press, 1921. Sir Orfeo, p 13-31. 